Proceedings of the 2011 Winter Simulation Conference

S. Jain, R. R. Creasey, J. Himmelspach, K. P. White, and M. Fu, eds.

\title{
A SMART SAMPLING SCHEDULING AND SKIPPING SIMULATOR AND ITS EVALUATION ON REAL DATA SETS
}

\author{
Claude Yugma \\ Stéphane Dauzère-Pérès \\ Jean-Loup Rouveyrol \\ École des Mines de Saint-Étienne, Centre Microélectronique de Provence, Site Georges Charpak \\ 880 Avenue de Mimet, Gardanne F-13541, France
}

\author{
Philippe Vialletelle \\ STMicroelectronics, 850 rue Jean Monnet \\ 38926 Crolles cedex, France
}

\author{
Jacques Pinaton \\ STMicroelectronics, Zone Industrielle Rousset \\ 13106 Rousset cedex, France
}

\author{
Christophe Relliaud \\ LFoundry, Zone industrielle Rousset \\ 84 Avenue Olivier Perroy, 13790 Rousset, France
}

\begin{abstract}
As modern manufacturing technology progresses, measurement tools become scarce resources since more and longer control operations are required. It thus becomes critical to decide whether a lot should be measured or not in order to get as much information as possible on production tools or processes, and to avoid ineffective measurements. To minimize risks and optimize measurement capacity, a smart sampling algorithm has been proposed to efficiently select and schedule production lots on metrology tools. This algorithm and others have been embedded in a simulator called "Smart Sampling Scheduling and Skipping Simulator" (S5). The characteristics of the simulator will be presented. Simulations performed on several sets of instances from three different semiconductor manufacturing facilities (or fabs) will be presented and discussed. The results show that, by using smart sampling, it is possible to drastically improve various factory performance indicators when compared to current fab sampling.
\end{abstract}

\section{INTRODUCTION}

Semiconductor manufacturing is a highly capital intensive venture, where maintaining competitiveness requires high efficiency, tight quality and reduction of cost. Among key factors to reach these objectives are control measurements and the sampling of lots. Indeed, the manufacturing process of a lot is long (more than 300 steps) and complex (re-entrant structure). It is therefore important to detect as quickly as possible defects, to avoid spending time and money to produce wafers that will be scrapped. Moreover, in modern semiconductor manufacturing facilities (fabs), the increase of the wafer size from $200 \mathrm{~mm}$ to $300 \mathrm{~mm}$ combined with the reduction in the size of the transistors lead to a sharp increase of the wafer cost. More complex production processes require the introduction of numerous controls, sometimes redundant (Williams, Gudmundsson, Monahan, and Shanthikumar 1999), at different manufacturing steps. It is thus important to know where controls should be performed and what type. Moreover, because metrology capacity is expensive and not infinite, not all lots can be measured. Hence, it is necessary to sample the lots that should be measured.

In the literature, some research has been conducted on static sampling which usually consists of selecting the lots to control in a systematic way, based on pre-specified rules (Lee, You, and Park 2002), (Lee 2002). 
Such a strategy may lead to missing critical lots that would drastically reduce risks if measured. However, its main advantage is the ease of its implementation. Another strategy, called dynamic sampling, is based on sampling a variable number of lots, where the sampling frequency position should be determined (Williams, Gudmundsson, Nurami, Stoller, Chatterjee, Seshadri, and Shantikumar 1999), (Lee, Lee, Liao, and Chang 2003), (Good and Purdy 2007). This approach is more efficient, since the number of sampled lots depends on the production state (Boussetta and Cross 2005). However, papers often do not specify how to select lots to be measured. Furthermore, it is difficult to use rules that are constantly changing. Finally, it is only very recently that smart sampling has been proposed where the best lots to measure are selected. Smart sampling consists in determining dynamically and in an intelligent way the best lots to sample for measurement. Constraints are taken into account, such as the capacity of measurement tools. The goal is to optimize some objective function such as the minimization of the risk. Smart sampling may include the possibility of skipping lots that are waiting to be measured, and the scheduling of lots on metrology tools. This type of studies is relatively recent; see (Holfed, Barlovic, and Good 2007), (Gershwin and Kim 2005), (Colledani and Tolio 2007) and (Dauzere-Peres, Rouveyrol, Yugma, and Vialletelle 2010).

In this paper, we first describe a prototype called "Smart Sampling Scheduling and Skipping Simulator" (S5). We then present and analyze the results obtained with S5 on real instances provided by three different semiconductor manufacturing facilities. A summary of the smart sampling algorithm and other sampling algorithms can be found in Section 2. The prototype with its characteristics is presented in Section 3. The numerical experiments are shown and discussed in Section 4. Section 5 concludes the paper and provides some directions for further research.

\section{SMART SAMPLING}

Let us recall that the problem consists in deciding which lots should be measured and in which order they should be measured on metrology tools. Three types of decisions must be taken:

- D1. Sample lots for measurement, i.e., decide whether a lot will continue directly to the next process step without inspection or should be measured and sent to the metrology tools.

- D2. Schedule lots on the metrology tools, i.e. assign and sequence lots on the metrology tools.

- D3. Skip lots in the metrology queue if necessary, i.e. avoid measuring a lot and send it to the next process step.

It must be noted that the order of decisions is not necessarily sequential, i.e., sample, schedule and finally skip. Decisions can be taken simultaneously, e.g. a lot may be skipped in the metrology queue and replaced by a lot that is sampled and brings more information than the skipped lot.

In the following, we describe the main features of the "Smart Sampling Scheduling and Skipping Simulator" (S5), and in particular the principles of the Global Sampling Indicator (GSI) algorithm.

\subsection{The Global Sampling Indicator (GSI) Mechanism}

Parts of this section can be found in (Dauzere-Peres, Rouveyrol, Yugma, and Vialletelle 2010). Decisions are taken based on the risks incurred by not measuring a lot. The aim is to sample lots in order to minimize an objective based on risks. To each lot is associated a risk array (same risk type for several tools and/or several risk types). This array contains the new value of each risk (or of the risk reduction) if the lot is measured. There are some warning and inhibit limits for each risk. Let us introduce the following notations:

- $\quad R$ : Number of risks,

- $W L_{r}$ : Warning limit for risk $r$,

- $I L_{r}$ : Inhibit limit for risk $r$,

- $R V_{r}$ : Current risk value for risk $r$,

- $G_{r, l}$ : Gain on risk $r$ if lot $l$ is measured, 
- $\quad N R V_{r, l}$ : New risk value $r$ if lot $l$ is measured, i.e. $N R V_{r, l}=R V_{r}-G_{r, l}$.

- $N R V_{r}(S)$ : New risk value if lots in set $\mathrm{S}$ are measured.

Our first implementation focuses on the defectivity area, where the risk value $R V_{r}$ corresponds to the Wafer at Risk (W@R) for production tool $r$. The W@R is the number of wafers processed on tool $r$ since the process of the latest lot measured in defectivity. This can be seen as the number of wafers which have been processed on tool $r$ since the latest good defectivity control. In this case, $G_{r, l}$ is the W@R reduction of tool $r$ if lot $l$ is measured. The W@R may only be reduced, i.e. $G_{r, l}$ can only be strictly positive if $t$ belongs to the set of tools on which lot $l$ has been processed.

Two control parameters are used. The Warning Limit $W L_{r}$ corresponds to the value of the W@R for production tool $r$ beyond which the situation starts to become critical. The Inhibit Limit $I L_{r}$ is the maximum number of wafers that can be run between two defectivity inspections for $r$. Specific action is needed in order to prioritize or facilitate the control of the considered tool. Otherwise, $I L_{r}$ will be reached and eventually production will be stopped on tool $r$.

Sampling a lot will be based on how much is gained when adding the lot to the set of lots already waiting to be measured. Since a risk array is considered, this is a multi-criteria decision problem. Moreover, depending on the metrology capacity, it might be interesting to skip a lot that is waiting to be measured to replace it with the new sampled lot. In this case, sampling a lot and choosing another lot to be skipped must be done simultaneously.

Hence, when a lot $l$ is available for measurement and a set $S$ of lots is currently waiting to be measured, it must be decided whether to include $l$ in $S$ and skip a lot $l^{\prime}$ in $S$, or to skip $l$. It is thus necessary to compare the impact on the risks of the following sets of lots: $S$ and $\left\{S-l^{\prime}\right\} \cup\{l\}, \forall l^{\prime} \in S$.

The Global Sampling Indicator (GSI) used to select the best lots for a given set of lots $S$ is defined below:

$$
G S I(S)=\sum_{r=1}^{R}\left(\frac{N R V_{r}(S)}{I L_{r}}\right)^{\alpha}
$$

The parameter $\alpha$ is used to put more or less emphasis on getting as far as possible from the inhibit limit for which the current risk value is closer. The set with the smallest GSI value is chosen. The GSI aims at selecting sets that contain lots that help to reduce risk values that are closer to their inhibit limits. The GSI has been extended to improve decisions when a W@R of a tool is far from its warning limit, but the new formula will not be presented in this paper.

\section{THE SMART SAMPLING SCHEDULING AND SKIPPING SIMULATOR}

In this section, we first present the algorithms that have been implemented in S5 and then describe its main characteristics.

\subsection{Sampling Algorithms}

1. The "Without Sampling or No Sampling" Algorithm. This algorithm gives the theoretical maximal values of the risk indicators.

2. The "All Sampling" algorithm. It consists in selecting all the lots that can be sampled, and considering infinite capacity on the metrology tools. There is no waiting time in front of the metrology tools but the measurement time of each lot is considered. It is important to note that this algorithm is used to determine theoretical minimal values of the risk indicators.

3. The "Threshold Sampling" algorithm. A lot 1 is only sampled when one of its associated W@R is above the warning limit and can be decreased, i.e. there is a production tool $r$ such that $R V r \geq W L r$ and $G_{r, l}>0$.

4. The "GSI sampling" algorithm. The mechanism presented in Section 2.1 is used to evaluate the impact of measuring all lots in the set, and to decide in real time whether a lot should be sampled, 
i.e. measured or not. It is important to note that the GSI is not only valid for one risk type (W@R in our first study) on multiple tools, but also for multiple risk types on multiple tools. For the reader who is interested to understand the the algorithm in more details, see (Dauzere-Peres, Rouveyrol, Yugma, and Vialletelle 2010).

5. The "Fab Sampling" algorithm. The lots that are sampled are the ones that are actually sampled in the historical data provided by the semiconductor manufacturers.

\subsection{Performance Indicators}

Several performance indicators are defined to measure the quality of a solution.

- Number of sampled lots: Number of lots that are chosen to be measured and which are placed in the metrology queue.

- Number of measured lots: Number of lots actually measured, i.e. the number of lots processed on a metrology tool.

- Number of skipped lots: Number of lots removed from the metrology queue, i.e. the number of lots that are sampled but not measured. This is due to the fact that these lots do no longer contribute to decrease the W@R of at least one tool, or are removed from the queue as they do not bring enough compared to other lots in the queue.

- Number of lots above a limit (warning or inhibit). This number should be as small as possible.

- Average W@R. This is the sum of the W@R of all production machines divided by the number

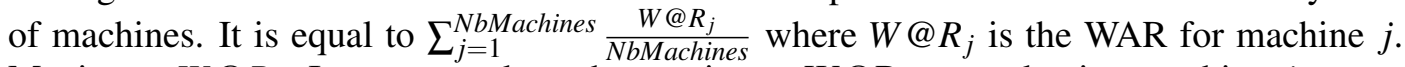

- Maximum $W @ R_{j}$. It corresponds to the maximum W@R on production machine $j$.

- Average Maximum W@R. This is the sum of the maximum W@ $\mathrm{R}$ of all production machines

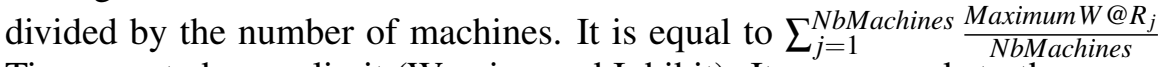

- Time spent above a limit (Warning and Inhibit). It corresponds to the sum of the times that all lots spent over the corresponding limit.

For more details of the performance indicators, see (Dauzere-Peres, Rouveyrol, Yugma, and Vialletelle 2010).

\subsection{Some Characteristics of S5}

The simulator has been implemented in Excel VBA. There is an input interface see Figure 1 where the parameters of the GSI algorithm and information on the metrology tools must be provided such as the average measurement time, the Warning and Inhibit limits for the tools, the number of metrology tools and the queue size. Note that different limits for each tool could be specified in a data sheet.

To perform a simulation, a file with historical data should be loaded with information on the start times of lots on production and metrology tools. Moreover, it is also necessary to have the control plans, i.e. what are the production operations that are validated by a metrology operation. Through a menu, it is possible to select the sampling algorithms to use before running a simulation. Some statistics and graphs are provided at the end of the simulation for all or a selected number of tools, and to compare the performance indicators between the selected sampling algorithms (see Figure 2 and Figure 3). In the Figure 2, the results of various indicators (lines) for different sampling algorithms (columns) are presented an actual instance simulation. The Figure 3 shows the changes in the WAR for two different machines. The behavior of these curves is close to sawtooth. The WAR increases (almost linearly) on each machine and as soon as a lot is measured, the corresponding WAR decreases abruptly. 


\section{improve

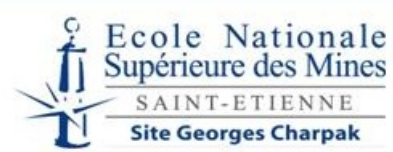

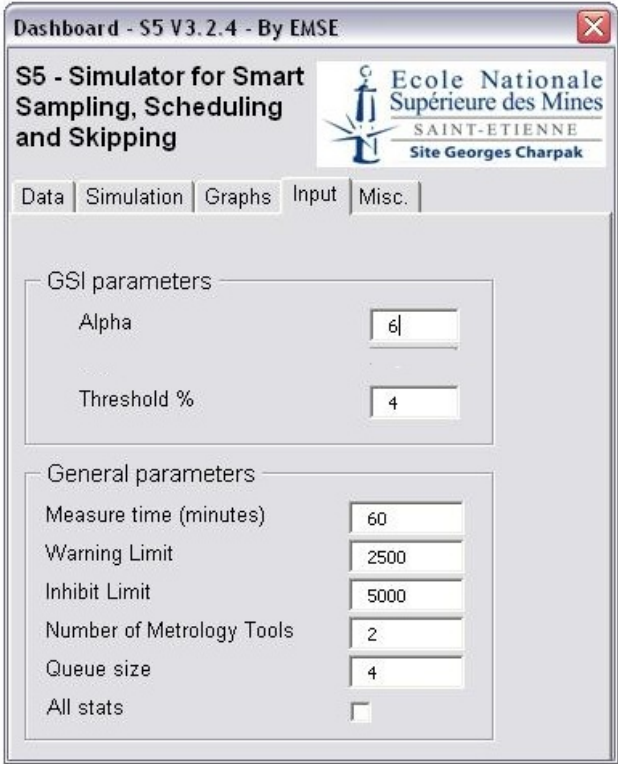

Figure 1: Main input parameters for S5.

\begin{tabular}{|c|c|c|c|c|c|c|c|c|}
\hline 4 & A & B & C & D & E & $\mathrm{F}$ & G & $\mathrm{H}$ \\
\hline 1 & & WithoutSampling & AllSampling & ThresholdSampling & IGSSampling & FabSampling & & \\
\hline 2 & Lots Sampled & 0 & 628 & 9 & 624 & 317 & & \\
\hline 3 & Lots Measured & 0 & 628 & 9 & 541 & 317 & & \\
\hline 4 & Lots Skipped & 0 & 0 & 0 & 80 & 0 & & \\
\hline 5 & Lots above Inhibit risk & 142 & 0 & 0 & 0 & 0 & & \\
\hline 6 & Lots above Warning risk & 498 & 0 & 34 & 0 & 39 & & \\
\hline 7 & Wafers above Inhibit risk & 3548 & 0 & 0 & 0 & 0 & & \\
\hline 8 & Wafers above Warning risk & 12418 & 0 & 850 & 0 & 975 & & \\
\hline 9 & Medium war (Average) & 1542,230769 & 136,2307692 & 909,3076923 & 140,1538462 & 332,4615385 & & \\
\hline 10 & Max war (Average) & 3030,615385 & 414,2307692 & 1941,461538 & 427,6923077 & 910,6153846 & & \\
\hline 11 & Time past with Warning risk (\%) & 7,009362536 & 0 & 0,308797187 & 0 & 0,61224537 & & \\
\hline 12 & Time past with Inhibit risk (\%) & 1,305498575 & 0 & 0 & 0 & 0 & & \\
\hline 13 & Simu execution Time & $00: 00: 00$ & $00: 00: 00$ & $00: 00: 00$ & $00: 00: 00$ & $00: 00: 00$ & & \\
\hline 14 & Stat execution Time & $00: 00: 00$ & $00: 00: 00$ & $00: 00: 00$ & $00: 00: 00$ & $00: 00: 00$ & & \\
\hline \multicolumn{9}{|l|}{15} \\
\hline 16 & alpha & & warning limit & Inhibit Limit & Threshold & measureTime & nbMetrologyTools & queueSize $\mathrm{Pr}$ \\
\hline 17 & 6 & & 2500 & 5000 & 4 & 0,041666667 & 2 & 4 \\
\hline
\end{tabular}

Figure 2: Some statistics provided by S5.

\section{EXPERIMENTS WITH S5 ON DATA FROM SEMICONDUCTOR MANUFACTURERS}

We present some of the experiments performed on data from ST Crolles, LFoundry and ST Rousset, respectively. The results show that, by using a smart sampling approach, and in particular the GSI sampling algorithm, it is possible to drastically reduce various performance indicators when compared to current fab sampling. 
Yugma, Dauzère-Pérès, Rouveyrol, Vialletelle, Pinaton, and Relliaud

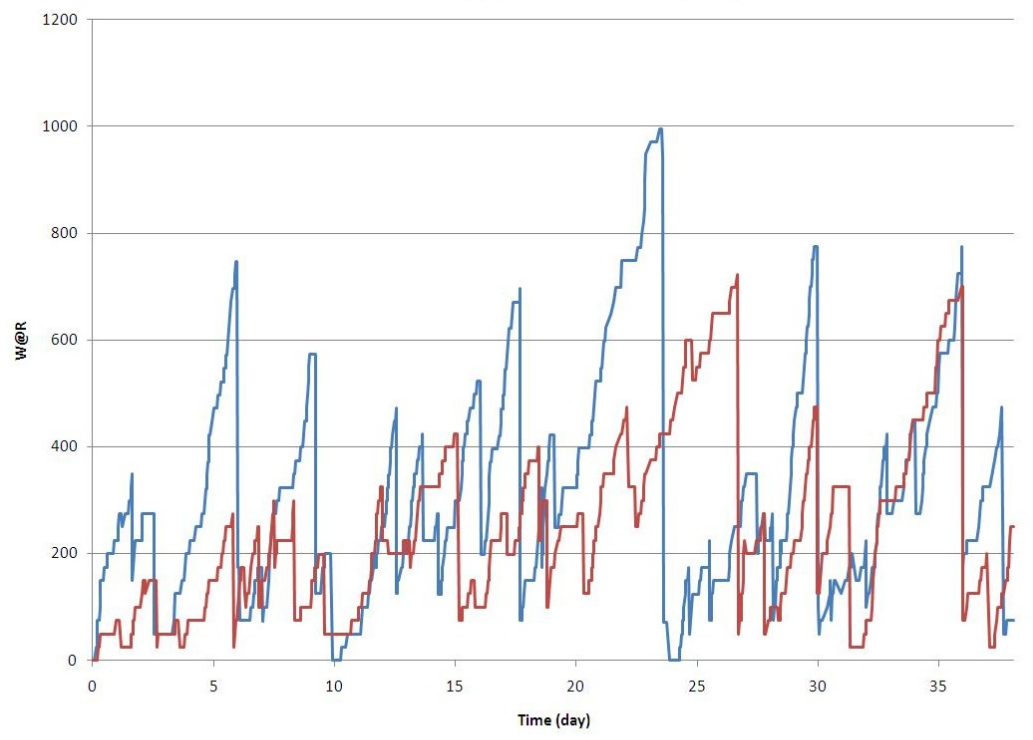

Figure 3: Some graphs provided by S5.

\subsection{Experiments on STMicroelectronics Crolles Data}

Table 1, 2 and 3 present the results on a set of data from ST Crolles. The following parameters are used for all tables: $\alpha=4, T H=4 \%$ and $I L=4000$ for all process tools. There are 3 metrology tools and the measurement time is 70 minutes. These values are chosen so that the metrology capacity is close to the actual capacity of the fab in number of measures on the planning horizon.

In Table 1, the Warning Limit WL for all tools is equal to 2000, i.e. half the Inhibit Limit IL, whereas it is equal to 3000 in Table 2 and 3500 in Table 3 respectively. The results show that all indicators can be greatly improved by using the GSI algorithm compared to the current fab sampling. Moreover, the threshold sampling algorithm is very sensitive to the value of the warning limits. Indeed, the results in Tables 2 and 3 show a strong degradation of the performances of the threshold algorithm, whereas the GSI algorithm is relatively robust.

Table 1: Results on data from STMicroelectronics Crolles (WL=2000, IL=4000).

\begin{tabular}{|c|c|c|c|c|c|}
\hline & $\begin{array}{c}\text { No } \\
\text { Sampling }\end{array}$ & $\begin{array}{c}\text { All } \\
\text { Sampling }\end{array}$ & $\begin{array}{c}\text { Threshold } \\
\text { Sampling }\end{array}$ & $\begin{array}{c}\text { GSI } \\
\text { Sampling }\end{array}$ & $\begin{array}{c}\text { Fab } \\
\text { Sampling }\end{array}$ \\
\hline No. of sampled lots & 0 & 9701 & 868 & 1143 & 467 \\
\hline No. of measured lots & 0 & 9701 & 375 & 437 & 437 \\
\hline No. of skipped lots & 0 & 0 & 493 & 706 & 0 \\
\hline Lots abobe Inhibit & 19697 & 39 & 66 & 64 & 7597 \\
\hline Lots above Warning & 29997 & 441 & 3369 & 2765 & 16395 \\
\hline Medium WAR (Avg.) & 2411 & 213 & 713 & 685 & 1262 \\
\hline Max. WAR (Avg.) & 4836 & 605 & 1623 & 1602 & 2821 \\
\hline Time above Warning (\%) & 2.61 & 0.04 & 0.25 & 0.20 & 1.27 \\
\hline Time above Inhibit (\%) & 1.27 & 0.00 & 0.00 & 0.00 & 0.48 \\
\hline
\end{tabular}


Yugma, Dauzère-Pérès, Rouveyrol, Vialletelle, Pinaton, and Relliaud

Table 2: Results on data from STMicroelectronics Crolles (WL=3000, IL=4000).

\begin{tabular}{|c|c|c|c|c|c|}
\hline & $\begin{array}{c}\text { No } \\
\text { Sampling }\end{array}$ & $\begin{array}{c}\text { All } \\
\text { Sampling }\end{array}$ & $\begin{array}{c}\text { Threshold } \\
\text { Sampling }\end{array}$ & $\begin{array}{c}\text { GSI } \\
\text { Sampling }\end{array}$ & $\begin{array}{c}\text { Fab } \\
\text { Sampling }\end{array}$ \\
\hline No. of sampled lots & 0 & 9701 & 413 & 998 & 467 \\
\hline No. of measured lots & 0 & 9701 & 260 & 434 & 467 \\
\hline No. of skipped lots & 0 & 0 & 153 & 564 & 0 \\
\hline Lots abobe Inhibit & 19697 & 39 & 39 & 39 & 7597 \\
\hline Lots above Warning & 24052 & 203 & 1511 & 502 & 10869 \\
\hline Medium WAR (Avg.) & 2411 & 213 & 906 & 665 & 1262 \\
\hline Max. WAR (Avg.) & 4836 & 605 & 2050 & 1605 & 2821 \\
\hline Time above Warning (\%) & 1.74 & 0.01 & 0.09 & 0.04 & 0.74 \\
\hline Time above Inhibit (\%) & 1.27 & 0.00 & 0.00 & 0.00 & 0.48 \\
\hline
\end{tabular}

Table 3: Results on data from STMicroelectronics Crolles (WL=3500, IL=4000).

\begin{tabular}{|c|c|c|c|c|c|}
\hline & $\begin{array}{c}\text { No } \\
\text { Sampling }\end{array}$ & $\begin{array}{c}\text { All } \\
\text { Sampling }\end{array}$ & $\begin{array}{c}\text { Threshold } \\
\text { Sampling }\end{array}$ & $\begin{array}{c}\text { GSI } \\
\text { Sampling }\end{array}$ & $\begin{array}{c}\text { Fab } \\
\text { Sampling }\end{array}$ \\
\hline No. of sampled lots & 0 & 9701 & 326 & 989 & 467 \\
\hline No. of measured lots & 0 & 9701 & 219 & 434 & 467 \\
\hline No. of skipped lots & 0 & 0 & 107 & 555 & 0 \\
\hline Lots abobe Inhibit & 19697 & 39 & 67 & 94 & 7597 \\
\hline Lots above Warning & 21682 & 119 & 1082 & 223 & 9034 \\
\hline Medium WAR (Avg.) & 2411 & 213 & 1008 & 666 & 1262 \\
\hline Max. WAR (Avg.) & 4836 & 605 & 2262 & 1597 & 2821 \\
\hline Time above Warning (\%) & 1.47 & 0.01 & 0.07 & 0.01 & 0.59 \\
\hline Time above Inhibit (\%) & 1.27 & 0.00 & 0.00 & 0.01 & 0.48 \\
\hline
\end{tabular}

\subsection{Experiments on LFoundry Data}

The results obtained on a set of data from LFoundry can be found in Table 4 . The following parameters are used: $\alpha=4, T H=4 \%, \mathrm{WL}=3000$ and IL $=5000$ for all process tools. There are 2 metrology tools and the measurement time is 70 minutes. The results show that, as in the case of ST Crolles, all performance indicators are improved with the GSI algorithm. Additional experiments, not presented here, show that the increase of the warning limits has the same negative effect on the threshold algorithm than with the ST Crolles data.

\subsection{Experiments on STMicroelectronics Rousset Data}

Tables 5 and 6 show the results on a set of data from ST Rousset. The following parameters are used: $\alpha=4, T H=4 \%, W L=2500$ and $I L=5000$ for all process tools. The measurement time is 60 minutes.

Table 5 corresponds to results with two metrology tools, and Table 6 to results with four metrology tools. It is interesting to see in Table 5 that, with half of the metrology capacity used in the actual fab sampling (52\%), the GSI algorithm provides results that are very good compared to the fab sampling. Table 6 shows that, by increasing the capacity closer to the actual fab sampling (82\%), the GSI algorithm is better than fab sampling on most performance indicators. Again, the threshold algorithm does not perform so well, and additional experiments, not presented here, show that it is very sensitive to the changes of the warning limits. Finally, note that, in this case, the GSI algorithm has little freedom to choose different lots 
Yugma, Dauzère-Pérès, Rouveyrol, Vialletelle, Pinaton, and Relliaud

Table 4: Results on data from LFoundry (WL=3500, IL=5000).

\begin{tabular}{|c|c|c|c|c|c|}
\hline & $\begin{array}{c}\text { No } \\
\text { Sampling }\end{array}$ & $\begin{array}{c}\text { All } \\
\text { Sampling }\end{array}$ & $\begin{array}{c}\text { Threshold } \\
\text { Sampling }\end{array}$ & $\begin{array}{c}\text { GSI } \\
\text { Sampling }\end{array}$ & $\begin{array}{c}\text { Fab } \\
\text { Sampling }\end{array}$ \\
\hline No. of sampled lots & $0 \%$ & $144 \%$ & $100 \%$ & $112 \%$ & $100 \%$ \\
\hline No. of measured lots & $0 \%$ & $144 \%$ & $84 \%$ & $95 \%$ & $100 \%$ \\
\hline Lots above Inhibit & $239 \%$ & $83 \%$ & $85 \%$ & $85 \%$ & $100 \%$ \\
\hline Lots above Warning & $168 \%$ & $86 \%$ & $92 \%$ & $91 \%$ & $100 \%$ \\
\hline Medium WAR (Avg.) & $313 \%$ & $95 \%$ & $97 \%$ & $97 \%$ & $100 \%$ \\
\hline Max. WAR (Avg.) & $270 \%$ & $91 \%$ & $93 \%$ & $93 \%$ & $100 \%$ \\
\hline Time above Warning (\%) & $237 \%$ & $81 \%$ & $90 \%$ & $89 \%$ & $100 \%$ \\
\hline Time above Inhibit (\%)\% & $382 \%$ & $80 \%$ & $83 \%$ & $83 \%$ & $100 \%$ \\
\hline
\end{tabular}

than the ones chosen in the actual fab sampling, since "All sampling" has only 7\% additional lots sampled and measured.

Table 5: Results on data STMicroelectronics Rousset, 2 metrology tools.

\begin{tabular}{|c|c|c|c|c|c|}
\hline & $\begin{array}{c}\text { No } \\
\text { Sampling }\end{array}$ & $\begin{array}{c}\text { All } \\
\text { Sampling }\end{array}$ & $\begin{array}{c}\text { Threshold } \\
\text { Sampling }\end{array}$ & $\begin{array}{c}\text { GSI } \\
\text { Sampling }\end{array}$ & $\begin{array}{c}\text { Fab } \\
\text { Sampling }\end{array}$ \\
\hline No. of sampled lots & $0 \%$ & $107 \%$ & $32 \%$ & $61 \%$ & $100 \%$ \\
\hline No. of measured lots & $0 \%$ & $107 \%$ & $31 \%$ & $52 \%$ & $100 \%$ \\
\hline Lots above Inhibit & $397 \%$ & $96 \%$ & $99 \%$ & $98 \%$ & $100 \%$ \\
\hline Lots above Warning & $301 \%$ & $99 \%$ & $108 \%$ & $104 \%$ & $100 \%$ \\
\hline Medium WAR (Avg.) & $356 \%$ & $97 \%$ & $107 \%$ & $102 \%$ & $100 \%$ \\
\hline Max. WAR (Avg.) & $303 \%$ & $97 \%$ & $106 \%$ & $102 \%$ & $100 \%$ \\
\hline Time above Warning (\%) & $317 \%$ & $99 \%$ & $108 \%$ & $104 \%$ & $100 \%$ \\
\hline Time above Inhibit (\%) & $395 \%$ & $98 \%$ & $100 \%$ & $99 \%$ & $100 \%$ \\
\hline
\end{tabular}

Table 6: Results on data STMicroelectronics Rousset, 4 metrology tools.

\begin{tabular}{|c|c|c|c|c|c|}
\hline & $\begin{array}{c}\text { No } \\
\text { Sampling }\end{array}$ & $\begin{array}{c}\text { All } \\
\text { Sampling }\end{array}$ & $\begin{array}{c}\text { Threshold } \\
\text { Sampling }\end{array}$ & $\begin{array}{c}\text { GSI } \\
\text { Sampling }\end{array}$ & $\begin{array}{c}\text { Fab } \\
\text { Sampling }\end{array}$ \\
\hline No. of sampled lots & $0 \%$ & $107 \%$ & $32 \%$ & $86 \%$ & $100 \%$ \\
\hline No. of measured lots & $0 \%$ & $107 \%$ & $32 \%$ & $82 \%$ & $100 \%$ \\
\hline Lots above Inhibit & $397 \%$ & $96 \%$ & $99 \%$ & $97 \%$ & $100 \%$ \\
\hline Lots above Warning & $301 \%$ & $99 \%$ & $108 \%$ & $101 \%$ & $100 \%$ \\
\hline Medium WAR (Avg.) & $356 \%$ & $97 \%$ & $107 \%$ & $99 \%$ & $100 \%$ \\
\hline Max. WAR (Avg.) & $303 \%$ & $97 \%$ & $105 \%$ & $99 \%$ & $100 \%$ \\
\hline Time above Warning (\%) & $317 \%$ & $99 \%$ & $107 \%$ & $100 \%$ & $100 \%$ \\
\hline Time above Inhibit (\%) & $395 \%$ & $98 \%$ & $99 \%$ & $98 \%$ & $100 \%$ \\
\hline
\end{tabular}

\subsection{Summary}

On all realized tests, the results on the ST Crolles data show that very large gains can be achieved on all risk indicators while measuring a smaller number of lots than in fab sampling. The results on the LFoundry data illustrate the same conclusions. The data from ST Rousset are a bit different from the others, since only $7 \%$ additional lots can be sampled and measured compared to fab sampling. Hence, with the same 
metrology capacity (i.e. number of lots that can be measured), the GSI algorithm has little flexibility to choose different lots than the ones chosen in fab sampling. This is why experiments were performed where the GSI algorithm has about half of the metrology capacity used in fab sampling. The obtained results show that the risk indicators are very close to the ones of fab sampling, and thus that metrology capacity could be saved with smart sampling. Also, all experiments show that the GSI algorithm is rather robust with regards to the critical input parameters that are Warning Limits and Inhibit Limits.

\section{CONCLUSION AND PERSPECTIVES}

In this paper, we presented a "Smart Sampling Scheduling and Skipping Simulator" (S5). It includes various sampling algorithms, and in particular a smart sampling algorithm called GSI (Global Sampling Indicator) algorithm that aims at sampling, scheduling and skipping lots based on the evaluation of the impact of measuring a set of lots. S5 has been implemented on Excel VBA and tested on actual defectivity data from three different fabs (STMicroelectronics Crolles, STMicroelectronics Rousset and LFoundry), showing significant gains on various performance indicators, in particular compared to how sampling is currently performed in the fabs.

Further studies on the means and variance of cycle time have to be done. An interesting research perspective for smart sampling is to consider the concept of Equipment Health Factor (EHF) which provides real-time information on the status of production tools. The objective is to extend our approach and S5 to integrate in smart sampling decisions the information from the EHF and the degree of confidence in the EHF model.

\section{ACKNOWLEDGEMENTS}

This work has been done under the sponsorship of the IMPROVE (Implementing Manufacturing science solutions to increase equiPment pROductiVity and fab pErformance) ENIAC European project.

\section{REFERENCES}

Boussetta, A., and A. Cross. 2005. "Adaptive Sampling Methodology for In-Line Defect Inspection". In Advanced Manufacturing Conference and Workshop, 25-31. Munich (Germany).

Colledani, M., and T. Tolio. 2007. "Impact of Quality Control on Production Systems Performance". Annals of the CIRP Manufacturing Technology 55 (1): 453-456.

Dauzere-Peres, S., J.-L. Rouveyrol, C. Yugma, and P. Vialletelle. 2010. "A Smart Sampling Algorithm to Minimize Risk Dynamically". In Advanced Semiconductor Manufacturing Conference, 307-310. San Francisco (USA).

Gershwin, S., and J. Kim. 2005. "Integrated Quality and Quantity Modelling of a Production Line”. OR Spectrum 27 (2-3): 287-314.

Good, R., and M. Purdy. 2007. “An MILP Approach to Wafer Sampling and Selection”. IEEE Transactions on Semiconductor Manufacturing 20 (4): 400-407.

Holfed, A., R. Barlovic, and R. Good. 2007. "A Fab-Wide APC Sampling Application”. IEEE Transactions on Semiconductor Manufacturing 20 (4): 393-399.

Lee, J. 2002. "Artificial Intelligence-Based Sampling Planning System for Dynamic Manufacturing Process". Expert Systems with Applications 22 (2): 117-133.

Lee, J., S. You, and S. Park. 2002. "A New Intelligence SOFM-based Sampling Plan for Advanced Process Control". Expert Systems with Applications 20 (2): 133-151.

Lee, S.-B., T.-Y. Lee, J. Liao, and Y.-C. Chang. 2003. "A Capacity-Dependence Dynamic Sampling Strategy”. In IEEE International Symposium on Semiconductor Manufacturing, 312-314.

Williams, R. D., K. Gudmundsson, K. Monahan, and J. Shanthikumar. 1999. "Optimized Sample Planning for Wafer Defect Inspection". In IEEE International Symposium on Semiconductor Manufacturing, 43-46. Santa Clara, CA (USA). 
Yugma, Dauzère-Pérès, Rouveyrol, Vialletelle, Pinaton, and Relliaud

Williams, R. D., K. Gudmundsson, R. Nurami, M. Stoller, A. Chatterjee, S. Seshadri, and J. G. Shantikumar. 1999. "Challenging the Paradigm of Monitor Reduction to Achieve Lower Product Costs". In Advanced Semiconductor Manufacturing Conference and Workshop, 420-425. Boston, MA (USA).

\section{AUTHOR BIOGRAPHIES}

CLAUDE YUGMA is Associate Professor at EMSE. He earned a PhD degree in Computer Science and Combinatorial Optimization at the Grenoble Institute of Technology, France. His research was initially focused on scheduling problems and consistency of global and local scheduling decisions in semiconductor manufacturing. He is now also working on the interactions between Advanced Process Control and scheduling and dispatching decisions in semiconductor manufacturing, with topics such as: dynamic sampling, preventive maintenance scheduling, etc. He was involved in the in the regional project Rousset 2003-2008 (with STMicroelectronics) and in the MEDEA+ European project HYMNE. Currently, he is involved in the ENIAC European Project IMPROVE. His email address is Yugma@emse.fr.

STÉPHANE DAUZÈRE-PÉRÈS is Professor at the Provence Microelectronics Center of the Ecole des Mines de Saint-Etienne, where he is heading the Manufacturing Sciences and Logistics Department. He received the Ph.D. degree from the Paul Sabatier University in Toulouse, France, in 1992; and his Habilitation á Diriger des Recherches from the Pierre and Marie Curie University, Paris, France, in 1998. He was a PostDoc Fellow at the Massachusetts Institute of Technology, U.S.A., in 1992 and 1993, and Research Scientist at Erasmus University Rotterdam, The Netherlands, in 1994. He has been Associate Professor and Professor from 1994 to 2004 at the Ecole des Mines de Nantes in France. He was invited Professor at the Norwegian School of Economics and Business Administration, Bergen, Norway, in 1999. Since March 2004, he is Professor at the Ecole des Mines de Saint-Etienne. His research mostly focuses on optimization in production and logistics, with applications in planning, scheduling, distribution and transportation. He has published more than 35 papers in international journals and 100 communications in conferences. His email address is Dauzere-Peres@emse.fr.

JEAN-LOUP ROUVEYROL is an engineer at EMSE after receiving its master degree from University of Avignon (France). He is involved in the ENIAC European Project IMPROVE. His email address is Rouveyrol@emse.fr.

PHILIPPE VIALLETELLE is an expert of the Operations and Methods System group at STMicroelectronics Crolles, France. He is responsible of one of the packages of the ENIAC European Project IMPROVE. After receiving an Engineering degree in Physics from the Institut National des Sciences Appliquees in 1989, he entered the semiconductor industry by working on ESD and physical characterization. His next experience was then in metrology before moving to process control. He finally integrated the Factory Integration world, through Industrial Engineering and is now responsible for the definition of methodologies and tools for the 300mm production line. His email address is Philippe.Vialletelle@st.com.

JACQUES PINATON is manager of the Process Control System Group at STMicroelectronics Rousset, France. After being an Engineer in metallurgy from Conservatoire National des Arts et metiers dAix en Provence, France, he joined ST in 1984. After five years within the process engineering group, he joined the Device Department to implement SPC, process control methodology, and tools. He participated in three generations of fab start up, and he is leading several Rousset Research and Development Programs on manufacturing science, such as automation, APC, and diagnostic. He is involved in the ENIAC European Project IMPROVE. His email address is Jacques.Pinaton@st.com.

CHRISTOPHE RELLIAUD is an engineer at LFoundry Rousset (France). He is involved in the ENIAC European Project IMPROVE. His email address is Christophe.Relliaud@lfoundry.com. 